\title{
The Status of Medical Devices and their Utilization in 9 Tertiary Hospitals and 5 Research Institutions in Uganda
}

By Robert Tamale Ssekitoleko', Beryl Ngabirano Arinda', Solomon Oshabahebwa' ${ }^{1}$, Lucy Kevin Namuli', Julius Mugaga ${ }^{1}$, Catherine Namayega', Emmanuel Einyat Opolot, Jackline Baluka, Charles Ibingira'2, lan Guyton Munabi², Moses Lutakome Joloba ${ }^{3}$

\footnotetext{
${ }^{1}$ Biomedical Engineering Unit, Department of Physiology, School of Biomedical Sciences, College of Health Sciences, Makerere University, Uganda

2 Department of Anatomy, School of Biomedical Sciences, College of Health Sciences, Makerere University, Uganda

${ }^{3}$ School of Biomedical Sciences, College of Health Sciences, Makerere University
}

\section{ABSTRACT}

Backgrounds and Objective: Advancements in technology have led to great strides in research and innovation that have improved healthcare provision around the world. However, the majority of the technology available is underutilized in SubSaharan Africa. In addition, the ever-increasing sophistication and cost of medical equipment means that access and proper use is limited in low- and middle-income countries (LMICs). There is, however, a general paucity of well-documented evidence for the utilization of medical equipment in LMICs. Therefore, this study evaluates the current availability and utilization of medical equipment in tertiary hospitals and research facilities in Uganda and provides baseline information to clinical/biomedical engineers, innovators, managers, and policymakers.

Material and Methods: The study evaluated the equipment currently used in 9 purposively selected public tertiary hospitals and 5 research laboratories representing different regions of Uganda. Data were collected by personnel specialized in biomedical engineering utilizing a mixed-method approach that involved inventory taking and surveys directed to the health workers in the designated health facilities.

Results: The hospitals contributed 1995 (85\%) pieces of medical equipment while the research laboratories contributed 343 (15\%) pieces amounting to 2338 pieces of equipment involved in the study. On average, $34 \%$ of the medical equipment in the health facilities was faulty, and $85.6 \%$ lacked manuals.

Discussion and conclusion: Although innovative solutions and donated equipment address the immediate and long-term goals of resource-constrained settings, our study demonstrated several issues around existing medical devices, and these need immediate attention.

Keywords - Medical Devices, Biomedical Engineering, Healthcare Technology, Clinical Engineering, Appropriate Healthcare Technologies, Health Technology Management.

Copyright (C) 2021. This is an open-access article distributed under the terms of the Creative Commons Attribution License (CC BY): Creative Commons - Attribution 4.0 International - CC BY 4.0. The use, distribution or reproduction in other forums is permitted, provided the original author(s) and the copyright owner(s) are credited and that the original publication in this journal is cited, in accordance with accepted academic practice. No use, distribution or reproduction is permitted which does not comply with these terms. 


\section{INTRODUCTION}

Advancements in technology have led to great strides in innovations and research, resulting in a general improvement in healthcare provision, greatly impacting diagnostics, monitoring, and therapy. Medical device technology has played a key role in preventing, diagnosing, treating, and rehabilitating many diseases and contributes to complex research and innovations such as understanding the entire human genome. ${ }^{1-3}$ Many innovative devices have been applied to the early diagnosis of complicated diseases, including non-communicable diseases such as cancer, and management of chronic illnesses such as diabetes and HIV/AIDS. They have also been utilized to invent and track many drug regimens for most deadly diseases such as tuberculosis and HIV. ${ }^{4-7}$ Access and effective use of healthcare technologies leads to improved quality of healthcare provision to most of the population worldwide. ${ }^{8,9}$ It is therefore essential to have functional equipment. This is particularly urgent in Sub-Saharan Africa, ${ }^{10-13}$ a region with $24 \%$ of the world disease burden, $1 \%$ of the global financial resources and $3 \%$ of the human resource capacity. ${ }^{14}$ Lack of functional equipment has a devastating effect on the quality of healthcare provision and research in resource-poor settings and affects the overall healthcare system. ${ }^{10,15}$

Many medical technologies have been made available to improve healthcare services in hospitals and research laboratories in Sub-Saharan Africa. ${ }^{16}$ Application of these technologies in service delivery ensures improved work efficiency and enhanced quality, leading to cost-effective medical care for patients. ${ }^{9}$ However, the availability of medical equipment does not necessarily translate to improve health service delivery in health facilities in low resource countries. ${ }^{17}$ Indeed, health institutions worldwide are still struggling with managing quality healthcare delivery in resource-constrained conditions. ${ }^{18}$ Most Sub-Saharan countries hugely depend on medical equipment donations to facilitate healthcare and research technology needs. ${ }^{19}$ In fact, nearly $80 \%$ of medical devices available in healthcare facilities in developing countries are donated or funded by international donors or foreign governments. ${ }^{20}$ Most of these devices are poorly maintained, under-utilized, and or out of service due to various reasons such as inaccessibility to spare parts, accessories, and consumables. ${ }^{21}$ In addition, the high rate of dysfunctional equipment is attributed to the rising costs of medical devices, lack of reliable power and water, lack of public infrastructure such as air-conditioned rooms, and inadequate planning. ${ }^{16,22}$ Perry and Malkin ${ }^{23}$ report that $38.3 \%$ of medical equipment in developing countries is non-functional because of the lack of trained professionals able to execute the needed repairs or maintenance, mainly biomedical engineering technicians (BMET) or biomedical engineers. ${ }^{23}$

Several approaches were been taken to spur innovations in contextually appropriate healthcare technologies to respond to the challenges outlined above. Funding mainly came from international donors and philanthropists. ${ }^{24}$ However, with the recent cessation or reduction of this funding due to shifting priorities such as the recent COVID-19 pandemic, most of these innovations have been abandoned due to the absence of sustainability plans. This has led to a setback in this field with actual progress showing little clinical efficiency. ${ }^{25,26}$ Apart from limited funding, WHO highlights other barriers that hinder the effective adoption of innovative solutions in low- and middle-income countries: inadequately trained support staff to manage the novel equipment, shortage of technical expertise, and designs not being suitable for the African setting. ${ }^{27}$

Steps have been taken to avert the challenges in medical equipment management, including designing novel medical equipment suitable for sub-Saharan Africa setting, ${ }^{28,29}$ developing policies on donations and equipment procurement, ${ }^{30}$ and training biomedical engineers locally to enhance technical support. ${ }^{31}$ There is little evidence published on how these efforts have improved medical equipment access to the people in most need.

Therefore, this paper aimed to evaluate the current availability, status, and utilization of medical equipment in tertiary hospitals and research facilitates in Uganda.

\section{METHODOLOGY}

\section{Study design and setting}

This was a cross-sectional study that utilized a mixedmethod approach that involved inventory taking and surveys. The study evaluated the equipment in current use in 9 purposively selected public tertiary healthcare facilities and five research laboratories to represent 
different regions of Uganda in January 2017. The research laboratories included three research-based institutions, that is, the microbiology laboratory at Makerere University College of Health Sciences (central region), the microbiology and molecular biology laboratories at Gulu University (Northern Region), and Mbarara University of Science and Technology (MUST) clinical and research laboratory (Western Region) and two independent research laboratories; Uganda Virus Research Institute (UVRI) and Infectious Disease Institute (IDI). Each of these laboratories serves as a reference laboratory receiving samples from neighboring hospitals for clinical diagnosis support and analyzing samples for research and academic purposes. Figure 1 below shows the spread of the facilities surveyed. The healthcare facilities selected serve about $21 \%$ of Uganda's Population, translating to about 7.9 million people as of 2017 Uganda population census estimates.

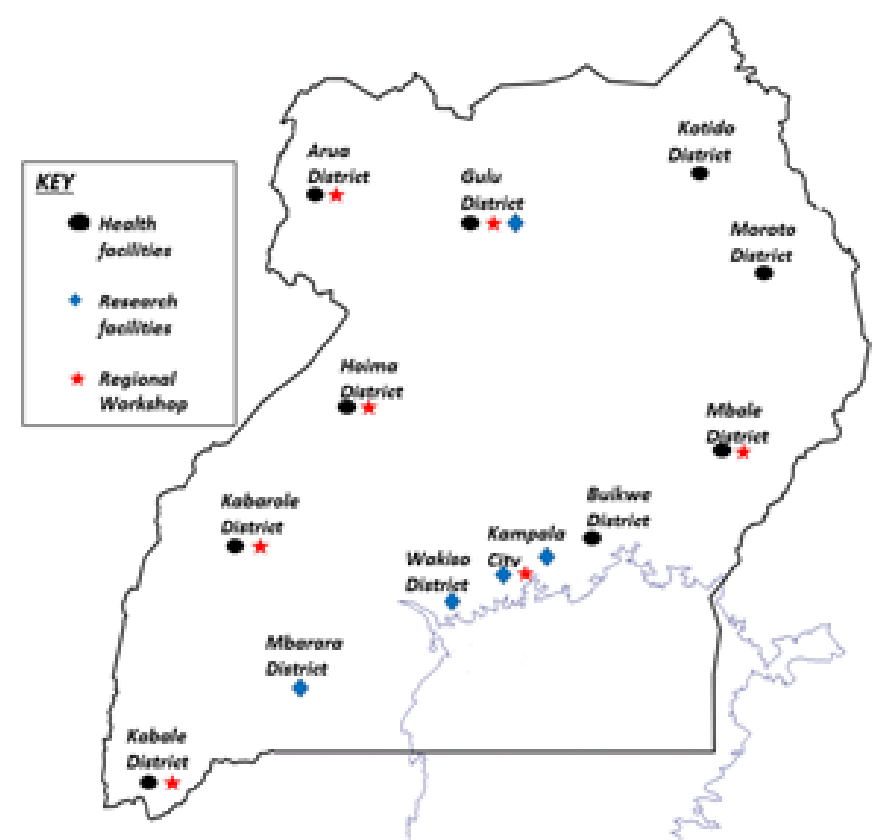

FIGURE 1. A map of Uganda showing the different locations of the study sites and nearby regional Biomedical Engineering workshops.

\section{Data collection and analysis}

All data collectors had a bachelor's degree in biomedical engineering and were given uniform data collection tools and were trained to conduct interviews and collect inventory. Data on the collection inventory was aimed at generating information on the working condition of the various devices currently available in the study facilities. The inventory assessment included all medical equipment available at the study site when conducting the study and excluded furniture, instruments, drugs, computers, computer accessories, and disposable tools and instruments. The equipment details collected in the inventory included the medical equipment name, type, model, equipment number, serial number, functionality, manufacturer, year of manufacturer, and location. The condition of the medical equipment was recorded using an A to $\mathrm{F}$ scale recommended by the Uganda Ministry of Health ${ }^{32}$ and the non-functional equipment in categories B, C, D, E, and F were further categorized as shown in Table 1.

TABLE 1. Key to the A-F Scale of Medical Equipment Conditions Used To Assess Medical Equipment in this Study

\begin{tabular}{|c|c|}
\hline Category & Interpretation \\
\hline $\mathrm{A}$ & Equipment in good working condition and in use \\
\hline $\mathrm{B}$ & Equipment in good working condition but not in use \\
\hline $\mathrm{C}$ & Equipment in use but need repair \\
\hline $\mathrm{D}$ & Equipment in use but needs replacement \\
\hline $\mathrm{E}$ & Equipment out of use but repairable \\
\hline F & Equipment out of use, to be disposed of \\
\hline
\end{tabular}

The data were analyzed using STATA version 14.0. Discrete variables were summarized by their means and standard deviations, whereas categorical variables were presented as frequencies and percentages. Logistic regression was used to compare the functionality of equipment between groups, and results were reported with odds ratios. All differences with a p-value less than 0.05 were considered statistically significant.

\section{Ethical consideration}

Ethical approval was obtained from Makerere University School of Biomedical Sciences Institutional Review Board and the Uganda National Council of Science and Technology (UNCST; \# SS 4166). Informed consent was obtained from all participants before enrolment into the study. Confidentiality was assured through de-identification of the data. 


\section{RESULTS}

This study included 2338 pieces of medical equipment categorized in 255 medical equipment types, of which the hospitals contributed $85 \%$ (1995). Table 2 shows the characteristics of the facilities included in the study. The out-patient department attendance per day characterized the study, number of admissions per day, population served, number of biomedical engineers and technicians (BMETs), number of pieces of medical equipment found, the percentage of medical equipment classified as nonfunctional, the number of pieces of equipment without manuals, and the number of manufacturers supplying the hospital with medical equipment. A total of 12 biomedical engineers and technicians (BMETs) were identified to be working in the hospitals studied, out of which only 3 had a bachelor's degree while the 9 were diploma holders. In the hospitals where the availability of manuals was recorded, more than $50 \%$ of the medical equipment had no manuals. On the other hand, the IDI research laboratory had manuals for all their equipment. The number of manufacturers was also relatively high, with the highest recorded being 120 manufacturers supplying a single hospital served by 2 BMETs.

TABLE 2. Characteristics of the Hospitals and Research Laboratories Included in the Study

\begin{tabular}{|c|c|c|c|c|c|c|c|c|}
\hline Health Facility & $\begin{array}{l}\text { Location } \\
\text { (Region in } \\
\text { Uganda) }\end{array}$ & $\begin{array}{c}\text { OPD } \\
\text { attendance } \\
\text { per day }\end{array}$ & $\begin{array}{c}\text { Admissions } \\
\text { per day }\end{array}$ & $\begin{array}{c}\text { Population } \\
\text { served }\end{array}$ & $\begin{array}{c}\text { No. of } \\
\text { BMETs } \\
\text { employed }\end{array}$ & $\begin{array}{l}\text { No. of pieces } \\
\text { of equipment } \\
\text { included (\% of } \\
\text { equipment non- } \\
\text { functional }{ }^{\star} \text { ) }\end{array}$ & $\begin{array}{l}\text { \% of } \\
\text { equipment } \\
\text { without } \\
\text { manuals }\end{array}$ & $\begin{array}{c}\text { No. of } \\
\text { manufacturers } \\
\text { recorded }\end{array}$ \\
\hline \multicolumn{9}{|l|}{$\begin{array}{l}\text { Regional Referral } \\
\text { Hospitals (RRH) }\end{array}$} \\
\hline Arua RRH & North Western & 443 & 65 & $\begin{array}{l}3.5 \\
\text { million }\end{array}$ & 1 & $200(50 \%)$ & $91 \%$ & 78 \\
\hline Fortportal RHH & Western & 254 & 70 & & 2 & $240(4 \%)$ & & \\
\hline Hoima RHH & Western & 413 & 65 & 3 million & 2 & $53(26 \%)$ & $77 \%$ & 31 \\
\hline Kabale RHH & South Western & 178 & 33 & 2 million & 2 & $510(30 \%)$ & & \\
\hline Mbale RHH & Eastern & 210 & 135 & & 1 & $347(36 \%)$ & & \\
\hline Mbarara RHH & South Western & 468 & 85 & 4 million & 2 & $392(52 \%)$ & $53 \%$ & 120 \\
\hline Moroto RHH & North Eastern & 160 & 20 & $\begin{array}{c}1.5 \\
\text { million }\end{array}$ & 1 & $13852 \%)$ & & 74 \\
\hline \multicolumn{9}{|l|}{ General hospitals } \\
\hline Kotido Hospital & North Eastern & 77 & 15 & & 0 & $59(54 \%)$ & $75 \%$ & 29 \\
\hline \multicolumn{9}{|l|}{ Health centre IV } \\
\hline Kawolo hospital & Central Region & 217 & 30 & $\begin{array}{c}1.2 \\
\text { million }\end{array}$ & 1 & $56(50 \%)$ & $68 \%$ & 25 \\
\hline \multicolumn{9}{|l|}{$\begin{array}{c}\text { Research } \\
\text { laboratories }\end{array}$} \\
\hline MUST & Western & & & & & $24(29 \%)$ & & 14 \\
\hline UVRI & Central & & & & & $153(29 \%)$ & & \\
\hline Makerere Univ. & Central & & & & & $23(22 \%)$ & & 11 \\
\hline Gulu Univ. & Northern & & & & & $114(10 \%)$ & $41 \%$ & 57 \\
\hline IDI & Central & & & & & $29(7 \%)$ & $0 \%$ & 20 \\
\hline
\end{tabular}

*All medical EQUIPMENT IN CONDITIONS B, C, D, E, F WERE CLASSIFIED AS NON-FUNCTIONAL. 
Table 2 shows the percentage of non-functional equipment was 4 to $54 \%$ with an average of $37 \%$, while the research laboratories had a better performance with the non-functional equipment ranging from 7 to $29 \%$ with a mean of $20 \%$. According to the A-F scale, further breakdown of the equipment categories (see Table 1) revealed that the non-functional medical equipment was spread out in categories B, C, and E, as shown in Table 3. Out of 2338 pieces of equipment, 157 were identified in category B, 296 in category C, and 193 identified in category E. The top reasons identified for equipment in category B were lack of user training and lack of consumables. On the other hand, the equipment in categories $\mathrm{C}$ and $\mathrm{E}$ was usually there because of a lack of spare parts, testing equipment to identify faulty equipment, and technical knowledge on performing repairs.

The medical equipment manufacturers were recorded at 6 of the health facilities and 4 of the research facilities. The number of manufacturers supplying the facilities ranged from 11 to 120 , with an average of 46 . This number varied with the number of pieces of medical equipment at each facility (Figure 2).

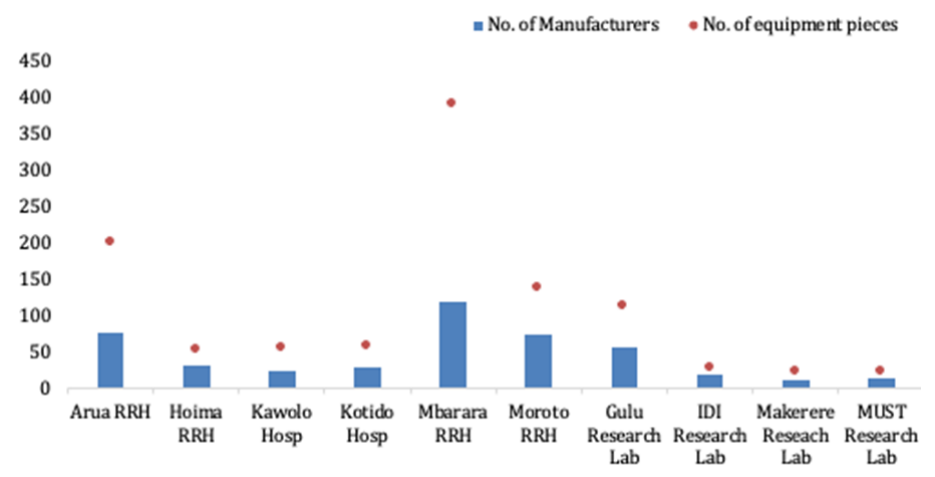

FIGURE 2. Bar graph showing variation in the number of equipment manufacturers supplying 6 of the 9 healthcare facilities and 4 of the 5 research facilities included in this study. The number corresponding to the highest point of each bar is the number of pieces of equipment in each facility and the number represented by the dot above each bar is the number of equipment pieces in that facility.

According to the study, 358 (15\%) pieces of equipment were donated and the facilities bought 195 (8\%) pieces of equipment. The remaining $77 \%$ of the equipment was classified as unknown because the interviewees could not ascertain whether they were donated or bought. The study also assessed the availability of user and technical manuals for the equipment and revealed that 345 (14.4\%) pieces of equipment had manuals, whereas 2055 (85\%) pieces of equipment had no manuals available (Table 2). Thus, $80 \%$ of the donated equipment had no manuals, whereas $86 \%$ of the equipment purchased had manuals.

Table 3 also shows the distribution of some of the equipment types identified in the six equipment categories. This table shows that a high number of oxygen concentrators and pipettes were found in category $C$. This was because the facilities did not have working oxygen sensors to determine the concentration of oxygen concentrators and lacked a clear basis for their use. Similarly, the pipettes were never or rarely calibrated. Many glucometers were not used despite being in good working conditions (category B) because of a lack of strips, while a large number of infant incubators and nebulizers in the same category were mainly due to lack of user training.

The non-functional equipment (categories B, C, D, $\mathrm{E}$, and F) was further categorized according to the host departments, and analysis revealed that dental and sterilization departments had the highest percentage of non-functional equipment. In contrast, the laboratory equipment in both laboratories within the hospitals and research laboratories had a significantly lower percentage of non-functional equipment than the mean. Indeed, when all pieces of laboratory equipment were excluded from analysis, the average percentage of non-functional equipment in the other departments increased from 37 to $47 \%$, as seen in Figure 3.

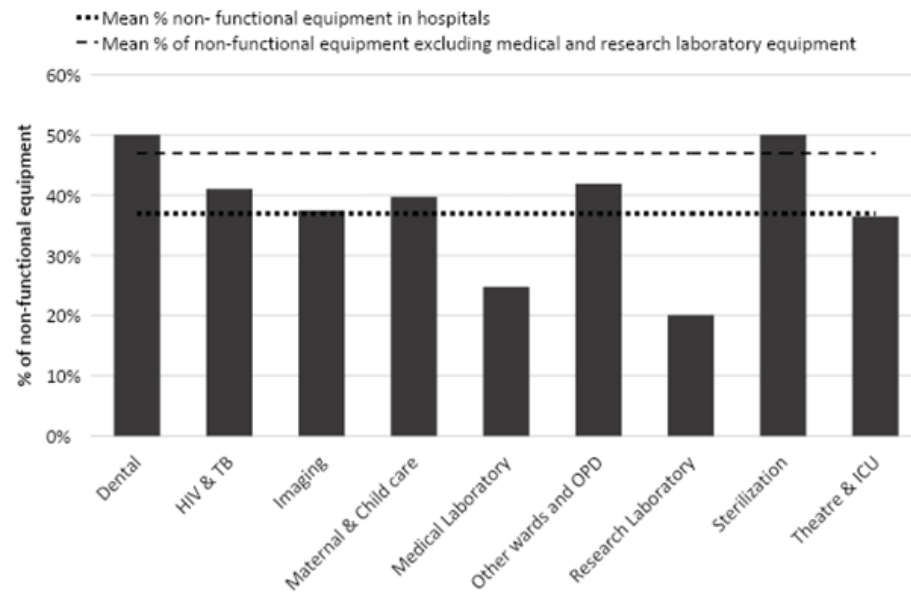

FIGURE 3. Percentage of non-functional equipment categorized according to the department, the mean percentage of non-functional equipment and then mean percentage of the non-functional equipment excluding medical and research laboratory equipment. 
TABLE 3. The Condition of Medical Equipment in the Hospitals, Health Facilities, and Top Medical Equipment Types Are Broken Down Into Each of the Six Categories in the A-F scale

\begin{tabular}{|c|c|c|c|c|c|c|c|}
\hline & \multicolumn{6}{|c|}{ Equipment condition categories } & \multirow{2}{*}{$\begin{array}{c}\text { Total \# of piece } \\
\text { of equipment }\end{array}$} \\
\hline & $\mathbf{A}$ & B & $\mathrm{C}$ & D & $\mathbf{E}$ & $\mathbf{F}$ & \\
\hline Total & $66 \%$ & $7 \%$ & $13 \%$ & $1 \%$ & $8 \%$ & $6 \%$ & 2,338 \\
\hline Hospitals & $63 \%$ & $7 \%$ & $13 \%$ & $1 \%$ & $9 \%$ & $6 \%$ & 1995 \\
\hline Research laboratories & $80 \%$ & $3 \%$ & $11 \%$ & $0 \%$ & $4 \%$ & $2 \%$ & 339 \\
\hline \multicolumn{8}{|l|}{ Medical Equipment Types } \\
\hline Refrigerator & $77 \%$ & $1 \%$ & $5 \%$ & $2 \%$ & $4 \%$ & $10 \%$ & 135 \\
\hline Weighing Scale & $58 \%$ & $5 \%$ & $9 \%$ & $2 \%$ & $18 \%$ & $9 \%$ & 126 \\
\hline Patient Monitor & $66 \%$ & $9 \%$ & $12 \%$ & $0 \%$ & $11 \%$ & $2 \%$ & 117 \\
\hline Suction Machine & $48 \%$ & $5 \%$ & $9 \%$ & $0 \%$ & $15 \%$ & $24 \%$ & 105 \\
\hline Autoclave & $53 \%$ & $8 \%$ & $19 \%$ & $1 \%$ & $8 \%$ & $10 \%$ & 98 \\
\hline Microscope & $76 \%$ & $7 \%$ & $8 \%$ & $1 \%$ & $6 \%$ & $3 \%$ & 90 \\
\hline Centrifuge & $76 \%$ & $1 \%$ & $4 \%$ & $1 \%$ & $1 \%$ & $17 \%$ & 78 \\
\hline BP Machine & $69 \%$ & $9 \%$ & $10 \%$ & $0 \%$ & $6 \%$ & $5 \%$ & 77 \\
\hline Oxygen Concentrator & $67 \%$ & $4 \%$ & $21 \%$ & $0 \%$ & $3 \%$ & $5 \%$ & 76 \\
\hline Operating Light & $57 \%$ & $3 \%$ & $10 \%$ & $13 \%$ & $11 \%$ & $6 \%$ & 63 \\
\hline Examination lamp & $59 \%$ & $17 \%$ & $7 \%$ & $0 \%$ & $9 \%$ & $9 \%$ & 46 \\
\hline Infant Incubator & $65 \%$ & $30 \%$ & $4 \%$ & $0 \%$ & $0 \%$ & $0 \%$ & 46 \\
\hline Pipette & $7 \%$ & $0 \%$ & $70 \%$ & $0 \%$ & $0 \%$ & $23 \%$ & 43 \\
\hline Freezer & $74 \%$ & $5 \%$ & $5 \%$ & $0 \%$ & $13 \%$ & $3 \%$ & 39 \\
\hline Anesthesia Machine & $62 \%$ & $11 \%$ & $14 \%$ & $0 \%$ & $8 \%$ & $5 \%$ & 37 \\
\hline Glucometer & $43 \%$ & $37 \%$ & $10 \%$ & $0 \%$ & $10 \%$ & $0 \%$ & 30 \\
\hline Operating Table & $83 \%$ & $3 \%$ & $7 \%$ & $7 \%$ & $0 \%$ & $0 \%$ & 30 \\
\hline Nebulizer & $39 \%$ & $25 \%$ & $14 \%$ & $0 \%$ & $18 \%$ & $4 \%$ & 28 \\
\hline Vortex Mixer & $88 \%$ & $0 \%$ & $8 \%$ & $0 \%$ & $0 \%$ & $4 \%$ & 24 \\
\hline Ultrasound Machine & $43 \%$ & $0 \%$ & $13 \%$ & $0 \%$ & $30 \%$ & $13 \%$ & 23 \\
\hline Hematology analyzer & $86 \%$ & $0 \%$ & $0 \%$ & $0 \%$ & $9 \%$ & $5 \%$ & 22 \\
\hline Ventilator & $17 \%$ & $11 \%$ & $6 \%$ & $0 \%$ & $67 \%$ & $0 \%$ & 18 \\
\hline
\end{tabular}

\section{DISCUSSION}

This study found that, on average, $37 \%$ of the medical equipment found both in the hospitals were not in use, needed repair, and was completely non-functional. These results agree with a similar study done in Southwest Ethiopia in 2016 that reported that $32.1 \%$ of healthcare equipment was broken. ${ }^{10,23}$ In addition, Malkin in 2011 reported that, on average, $40 \%$ of medical equipment in resource-constrained countries is out of service. ${ }^{23}$ Our studies showed that the percentage of non-functional equipment in research laboratories was reduced to $20 \%$. Strategies identified that led to this improvement included (1) the provision of technical and user manuals. The IDI 
research laboratories had manuals for all their medical equipment, whereas all the hospitals surveyed did not have manuals for more than $50 \%$ of their equipment, implying a lack of technical support in proper use, maintenance, and repair; and (2) the laboratory equipment in both hospitals and research laboratories were supplied with service contracts usually from a local distributor. The distributor is tasked with user training, regular preventive maintenance (usually on a 6-month basis), and corrective maintenance upon breakdown. In general, medical equipment in the other department was procured with hardly any plans for their service and maintenance during their life span; (3) Research laboratories usually have funds to support medical equipment maintenance and repair.

It was observed that $7 \%$ of medical equipment was in good condition but out of service. These results fall within the same range as another study conducted in Ethiopia, which found the frequency of equipment in good condition but not in use ranging from 3 to $21 \%$, with a mean of $12 \% .{ }^{10}$ An example we saw in our study was glucometers, which have a huge potential in the fight against diabetes by providing fast and affordable point-of-care blood glucose measurement in low-resource settings. While the devices are cheap, the glucometer strips are unaffordable for many patients in low-resource settings thus are never used. Other reasons for not putting functional equipment to use included not knowing how to use the equipment correctly, lack of installation space, and lack of required infrastructure and utilities. ${ }^{21}$

The results also show that $13 \%$ of the medical equipment identified in the health facilities was faulty but used on patients. For example, we found some oxygen concentrators in use but delivered oxygen concentrations as low as $45 \%$ compared to the recommended concentrations greater than $82 \% .{ }^{33}$ This was often due to a lack of the right tools or testing equipment for the equipment functionality. There were also cases where the users were aware that the equipment was faulty but used it due to a lack of alternative options. This was usually coupled with a lack of spare parts and technical personnel and insufficient funds to support corrective maintenance. The use of faulty equipment on patients affected the overall outcomes in diagnosis and therapy. ${ }^{34}$
FACTORS AFFECTING MEDICAL EQUIPMENT UTILIZATION

\section{Medical equipment management}

This study found that hospitals did not have manuals for $68 \%$ of their medical equipment. Logistic regression analysis showed that the lack of equipment manuals was statistically related to medical equipment being non-functional ( $p$-value $<0.001$ ). Without these guides, equipment maintenance becomes very difficult, especially in Uganda, where nearly all medical equipment is imported with limited contact with the manufacturers. A survey conducted by the ministry of health in 2015 supported these findings, which found that only $13.4 \%$ of the health facilities in Uganda had scheduled medical equipment maintenance and that only $37 \%$ of the health facilities in Uganda have a budget for routine maintenance and repair of medical equipment. ${ }^{35}$ This failure to follow routine maintenance procedures results in the escalation of equipment faults. Therefore, collective efforts from medical equipment manufacturers, local distributors, health facilities, and the ministry of health are essential to provide the technical and user guides for medical equipment, put measures in place to provide technical support, source and avail funding for medical equipment management, and carry out routine user training and preventive maintenance.

\section{Technical human resource}

Our results show that in each of the hospitals included in the study, one or two BMETs, was responsible for maintaining and repairing the medical equipment at the health facility. When this workload is compared with the number of pieces of equipment identified and the number of manufactures supplying each hospital, on average, each BMET was charged with maintaining 167 pieces of equipment and from 51 variant manufacturers, each supplying a unique model of medical equipment. In addition, the BMETs in the regional referrals were expected to maintain the medical equipment in the lower-level health facilities. With little funding, lack of spare parts, manuals, and limited technical support from the manufacturers, these BMETs are indeed overwhelmed.

There have been considerable efforts to train BMETs locally; as of March 2021, seven teaching institutions train biomedical engineers and technicians at various levels. Makerere University, which pioneered bachelor's training 
for Biomedical Engineers in Uganda, has graduated 150 biomedical engineers at bachelor's level. However, the level of uptake by the Ministry of Health into the public health care system has been low. This is reported to be due to limited financial recourses. In addition, inadequate personnel available in health facilities to guide the procurement process, train users, and conduct routine maintenance and repair of medical equipment significantly contributes to medical equipment failure..$^{31,36}$

\section{Administrative support}

Administrative structures play a crucial role in medical equipment management. This can explain the variations observed in the percentage of non-functional equipment among the health facilities in this study. For example, most research laboratories have autonomous or semi-autonomous administrative structures and considerable donor funding that enable fast procurement of the required spare parts, consumables, and contracting skilled human resources to increase medical equipment utilization. ${ }^{37}$ Public hospitals, on the other hand, are characterized by long bureaucracies in the procurement process and minimal funding to support medical equipment maintenance. ${ }^{38}$ Some hospitals have, however, streamlined their procurement process to support infrastructural and resource utilization. The Biomedical Engineering workshop in one of the hospitals, for example, operates with a framework contract in which a comprehensive list of spare parts and consumables is submitted to procurement at the beginning of each financial year, and the items are purchased in a batch. ${ }^{39}$ This, therefore, eases repairs of medical equipment that require spare parts previously identified and listed. Thus, practical approaches to abridge convoluted administrative procedures to enhance infrastructural and resource utilization are paramount to improving medical equipment utilization in health facilities in low-resource settings.

\section{Procurement guidelines}

Despite recommendations by $\mathrm{WHO}^{30}$ and Ministry of Health $^{40}$ to regulate donated equipment, many hospitals in Uganda still accept medical equipment donations without following the guidelines to ensure that the equipment is fit for purpose and the setting. ${ }^{41}$ Additionally, there is still a lack of adequate procurement tools to assist hospital administrators in the appraisal of new equipment before purchasing in low-resource settings. ${ }^{42}$ This primarily contributed to the observed $7 \%$ of medical equipment being purchased or donated but never put to use due to lack of installation space, lack of consumables, or incompatibility with existing infrastructure and resources in this study. Additionally, it seemed that hospitals were eager to acquire medical equipment at low initial costs without considering the lifetime / hidden costs of the medical equipment, such as cost of consumables, maintenance costs, and cost of required utilities, among others. It was observed that medical equipment suppliers commonly offer health facilities medical equipment at low or no cost but charge them highly to procure reagents and consumables over long periods. However, the equipment procured under this contract ends up unused as the hospitals and patients cannot afford the cost of reagents. Another example observed was sterilization equipment procured by the hospital, but it later realized that the equipment's electricity consumption was way above the hospital's budget, thus putting it out of use. These point to deficiencies in the procurement appraisal process and a lack of technical guidance during procurement.

\section{Supporting infrastructure and resources}

System-wide deficiencies in infrastructure and resources to support medical technologies in low-resource countries have been shown to affect the utilization of medical devices. The lack of clean water, stable electricity supply, space, and administrative structures also affects medical equipment utilization, especially in the lower level health facilities and facilities in hard-to-reach areas. For example, some of the equipment identified in the study was designed for use with a $110 \mathrm{~V}$ power supply, and yet Uganda has a $240 \mathrm{~V}$ power supply. Without a step-down transformer, this equipment will remain unused in category B for years. Another example is autoclaves designed to operate with distilled water, yet the hospitals struggle to get access to distilled water. These are thus used with ordinary tap water, which significantly reduces their lifespan. This is, therefore, vital to consider in the design of novel medical equipment or during the procurement process.

\section{Innovations and implications}

Novel approaches custom-made to suit low resource settings provide an alternative to the hugely dependent 
on donated equipment. Development of these innovative inventions have been supported and financed by international donors and philanthropist. ${ }^{25}$ However, the majority of the funding comes to an end, and the inventions are abandoned ${ }^{26}$ while those that progress fails to translate to the African setting. The WHO highlighted that one significant barrier to the effective adoption of these inventions is that the design technology incorporated in these innovations does not suit the African setting. ${ }^{28}$ This study showed that most unused equipment in category B was new technologies that could not be applied to the Ugandan setting. An example was the microscopes; contributing to the $7 \%$ equipment in category $\mathrm{B}$ were new microscopes in storage because of inadequately trained support staff to manage the novel equipment while other models applied sophisticated technologies that were not suitable for the Ugandan settings. These findings align with WHO findings on the reasons for hindrances to the effective adoption and utilization of innovations in lowand middle-income countries. ${ }^{28}$ There is, therefore, a need for more emphasis on the context when designing new technologies for low- and middle-income countries.

\section{CONCLUSION}

Although innovative solutions and donated equipment address the immediate and long-term goals of resourceconstrained settings, our study showed that most of this equipment does not translate to the African setting, with an average of $37 \%$ of the equipment in hospitals non-functional. Research laboratories have successfully reduced non-functional equipment to $20 \%$ by ensuring that medical equipment is supplied with manuals and technical assistance, negotiating service contracts with the distributors, and securing funding for medical equipment management. Other factors noted as affecting medical equipment utilization include medical equipment management, technical human resource, administrative support, procurement procedures, supporting infrastructure, and resources.

\section{GRANT SUPPORT}

This research was funded by the NIH Forgarty International Center under grant number 1D71TW010337-01. The funder provided funds as part of a large study and did not contribute in the design of this study and collection, analysis, and interpretation of the data or writing of the manuscript.

\section{REFERENCES}

1. Ventola CL. Challenges in evaluating and standardizing medical devices in health care facilities. PT 2008;33(6):348-59.

2. Meyerson M, Gabriel S, Getz G. Advances in understanding cancer genomes through second-generation sequencing. Nature Reviews Genetics 2010;11:685-96.

3. Morozova 0, Marra MA. Applications of next-generation sequencing technologies in functional genomics. Genomics 2008;92(5):252-64.

4. Kwan C and Ernst JD. HIV and tuberculosis: A deadly human syndemic. Clinical Microbiology Reviews 2011;24.

5. Samb, B. et al. Prevention and management of chronic disease: A litmus test for health-systems strengthening in low-income and middle-income countries. The Lancet 2010:376.

6. Beaglehole, R. et al. Improving the prevention and management of chronic disease in low-income and middle-income countries: a priority for primary health care. The Lancet 2008;372.

7. Atun, R. et al. Improving responsiveness of health systems to non-communicable diseases. The Lancet $2014 ; 381$.

8. Otero HJ, Ondategui-Parra S, Nathanson EM, et al. Utilization management in radiology: basic concepts and applications. J Am Coll Radiol 2006;3.

9. Donabedian. A. Evaluating the quality of medical care. Milbank Quarterly 2005;83.

10.Ademe BW, Tebeje B, Molla A. Availability and utilization of medical devices in Jimma zone hospitals, Southwest Ethiopia: A case study. BMC Health Serv Res 2016;16.

11.Kim JY, Farmer P, Porter ME. Redefining global healthcare delivery. The Lancet 2013;382.

12. Meara JG, et al. Global Surgery 2030: Evidence and solutions for achieving health, welfare, and economic development. The Lancet 2015;386. 
13.Elbireer AM, Opio AA, Brough RL, et al. Strengthening public laboratory service in sub-Saharan Africa: Uganda case study. Lab Med 2011;42.

14.Anyangwe SCE and Mtonga C. Inequities in the global health workforce: The greatest impediment to health in Sub-Saharan Africa. Int J Environment Res Public Health 2007;4.

15. Kiwanuka SN, et al. Access to and utilisation of health services for the poor in Uganda: a systematic review of available evidence. Trans Royal Soc Trop Med Hygiene 2008;102.

16. Bryce CL and Clin KE. The supply and use of selected medical technologies. Health Aff 1998;17.

17. Howie SRC, et al. Beyond good intentions: Lessons on equipment donation from an African hospital. Bull. World Health Organ 2008;86.

18. Odedra M, Bennett M, Goodman S, and Lawrie M. SubSaharan Africa: A technological desert. Communicat ACM 1993;36.

19.Dohn MN and Dohn,AL. Quality of care on short-term medical missions: experience with a standardized patient record and related issues. Missiology An Int. Rev 2003;31.

20.World Health Organization. Medical device donations: considerations for solicitation and provision. WHO Med. Device Tech Ser 2011.

21.Emmerling D, Dahinten A, and Malkin RA. Problems with systems of medical equipment provision: an evaluation in Honduras, Rwanda and Cambodia identifies opportunities to strengthen healthcare systems. Health Technol (Berl) 2018;8.

22. Malkin RA. Design of health care technologies for the developing world. Ann Rev Biomed Engineer 2007;9.

23.Perry L and Malkin R. Effectiveness of medical equipment donations to improve health systems: How much medical equipment is broken in the developing world? Med Biol Engineer Comput 2011;49.

24. Masum H. et al. Venture funding for science-based African health innovation. BMC Int Health Hum Rights 2010;10.
25.Adelman HS and Taylor L. On sustainability of project innovations as systemic change. J Educat Psychol Consult 2003;14.

26. Martelli N and van den Brink H. Special funding schemes for innovative medical devices in French hospitals: The pros and cons of two different approaches. Health Policy (New. York) 2014;117.

27.World Health Organization. Medical Devices : Managing the mismatch: an outcome of the priority medical devices project: methodology briefing paper. World Heal. Organ 2010.

28. Richards-Kortum R and Oden M. Devices for lowresource health care. Science 2013;342.

29.WHO. Compendium of innovative health technologies for low-resource settings: assistive devices, eHealth solutions, medical devices. WHO Library Cataloguingin-Publication Data; 2014.

30.World Health Organization. Guidelines for Health Care Equipment Donations; 1997.

31.Ploss B. et al. Part II: U.S.-Sub-Saharan Africa Educational Partnerships for Medical Device Design. Ann Biomed Eng 2017;45.

32.Ministry of Health. Operation Manual for Regional Medical Equipment Maintenance Workshops and Medical Equipment Maintenance Guidelines A; 2013.

33.WHO. Technical Specifications for Oxygen Concentrators. WHO Medical Device Technical Series; 2015.

34. Mosadeghrad, A. M. Factors influencing healthcare service quality. Int J Heal Policy Manag 2014;3.

35. Ministry of Health. Health Sector Development Plan 2015/16 - 2019/20. RoU 110; 2015.

36. Malkin R and Keane A. Evidence-based approach to the maintenance of laboratory and medical equipment in resource-poor settings. Med Biol Eng Comput 2010;48.

37.Uthman OA, et al. Increasing the value of health research in the WHO African Region beyond 2015 - Reflecting on the past, celebrating the present and building the future: A bibliometric analysis. BMJ Open 2015;5.

38.Sekyonda Z, et al. Supply chain of routine orthopaedic implants in Kampala, Uganda: public-private 
workarounds arising from poverty and scarcity. Glob Heal Innov 2018;1.

39.Auditor General. Management of Procurement and Distribution of Essential Medicines and Health Supplies by National Medical Stores. 2016. Available at: http://www.oag.go.ug/wp-content/uploads/2017/04/ Procurement-Distribution-of-Essential-MedicinesHealth-Supplies-by-NMS.pdf. (Accessed: 18th February 2021)
40.Ministry of Health. National Medical Equipment Policy. 2009.

41. Auditor General. Report of the on Acquisition and Utilization of Medical Equipment Under Uganda Health Systems Strengthening Project (UHSSP). 2015.

42.Houngbo PT, et al. The root causes of ineffective and inefficient healthcare technology management in Benin public health sector. Heal Policy Technol 2017;6. 